\title{
Influence of electromagnetic effect parameters on ferromagnetic powders fluidity
}

\author{
Ivan N. Egorov ${ }^{1, *}$, and Nikolay Ya. Egorov ${ }^{2}$ \\ ${ }^{1}$ Don State Technical University, 344000 Rostov-on-Don, Russian Federation \\ ${ }^{2}$ Southern Federal University, 344006 Rostov-on-Don, Russian Federation
}

\begin{abstract}
The paper presents result of experimental studies of ferromagnetic powders fluidity management by effecting on particulate medium with constant and alternating gradient magnetic fields. Induction lines of constant magnetic field are perpendicular to symmetry axis of powder hopper. Alternating field is created by electromagnet with one tapering pole and has higher induction gradient values near hopper outlet. These fields form magneto fluidized bed from powder in hopper allowing powders of barium and strontium hexaferrite to flow through the hopper outlet. Dependencies of efflux speed from parameters of electromagnetic fields are presented for barium hexaferrite powder with average particle size $1 \mu \mathrm{m}$ and strontium hexaferrite powders with average particle sizes 1 and $9 \mu \mathrm{m}$.
\end{abstract}

\section{Introduction}

Ferromagnetic powders possess tendency to particle aggregation [1-4] that influences their behaviour during mixing, press-moulds filling, moulding etc. In ferromagnetic powders production and their application, fluidity becomes important technological characteristic [5-7]. Various methods of stimulated fluidity formation are applied to intensify the process of powder transportation. Hoppers can be supplied with vibrators, rippers, rotors of various constructions [8,9]. Fluidization by magnetic fields application is effective in case of ferromagnetic powders [10-12]. Powder fluidity depends on its density, humidity, particle size distribution, specific surface area, form and condition of particle surface etc. [13]. The main cause preventing stable powder efflux from outlet is particle aggregation and formation of arched structures [14]. Effective method of stimulated fluidity formation of fine magnetic powders is the effect on particulate medium by alternating gradient and constant magnetic fields with mutually perpendicular induction lines [15-17]. Variation of fields configuration and parameters of electromagnetic effect on particulate medium allows varying structuralmechanical properties and its rheological properties depending on specific technological requirements.

To establish regularities of stimulated fluidity process and conditions of stable powder efflux through thin outlet, it is necessary to find out the relationship between powder rheological characteristics and electromagnetic fields effecting on it.

* Corresponding author: egorovivan1@gmail.com 


\section{Problem}

Lack of natural fluidity doesn't allow press-mould filling with powders of magnetic materials without applying external fluidizing effect. Particle aggregation that prevents steady efflux of powder can be reduced by various methods. Electromagnetic effect is the most effective in case of magnetohard powders though its efficiency depends from parameters of applied fields. The goal is to determine regimes of electromagnetic effect on powders of barium and strontium hexaferrite that allow to obtain high and steady efflux speed and thereby precise press-mould filling.

\section{Experiment}

To study regularities of stimulated fluidity formation through small outlets we chose strontium and barium hexaferrite powders without natural fluidity. Changing of rheological characteristics of particulate system was processed by effect of constant magnetic field with induction $\mathrm{Bc}$ and alternating gradient magnetic field with induction gradient $\mathrm{dB} / \mathrm{dy}$. The speed of mass efflux $(v, \mathrm{mg} / \mathrm{s})$ through $2 \mathrm{~mm}$ diameter outlet was measured at various electromagnetic effect regimes. Arithmetic mean of 5 measurements was taken as result of mass efflux speed.

Scheme of the device (Fig. 1) for study of electromagnetic effect influence on powder fluidity formation includes alternating current electromagnets (1), constant current electromagnets (2), hopper (3) with mesh (4), powder receiver (5). The location of alternating current electromagnet poles (Fig. 1) provides vertical induction lines of alternating gradient magnetic field. Tapering low electromagnet pole provides higher induction gradient in the area below hopper outlet. Constant magnetic field induction lines are perpendicular to induction lines of alternating magnetic field. Mesh with $1 \mathrm{~mm}$ size of cell is located above the outlet eliminating its blockage by large particles.

The hopper was filled with defined powder mass. Both electromagnets were turned on for a fixed time. Powder, effected by magnetic fields, poured out through the outlet in the receiver. The mass of poured out powder was measured by electronic balance.

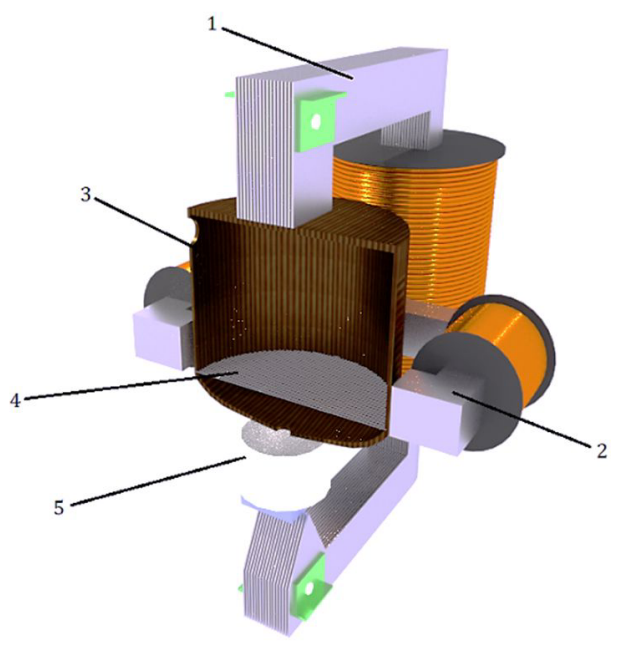

Fig. 1. Scheme of the device for studying electromagnetic effect of magnetohard powders fluidity: 1 - alternating current electromagnet; 2 - constant current electromagnet; 3 - hopper with powder; 4 - mesh with $1 \mathrm{~mm}$ cell size; 5 - powder receiver. 
External magnetic field seeks to align magnetic particles along the direction of resulting induction vector. Besides, particles are affected by local magnetic fields of surrounding particles. In alternating gradient magnetic field, the direction of force effecting on structure elements formed from powder particles, changes on the opposite with frequency $50 \mathrm{~Hz}$. At certain parameters of electromagnetic effect, the powder on mesh in hopper becomes fluidized forming dynamically steady weighted state of particulate medium $[15,18]$. Varying parameters of electromagnetic effect one can change the intensity of structural elements mobility in the magneto fluidized bed. Processes of aggregate destruction and formation of secondary aggregates $[19,20]$ take place in the fluidized bed influencing on the speed of mass efflux from outlet.
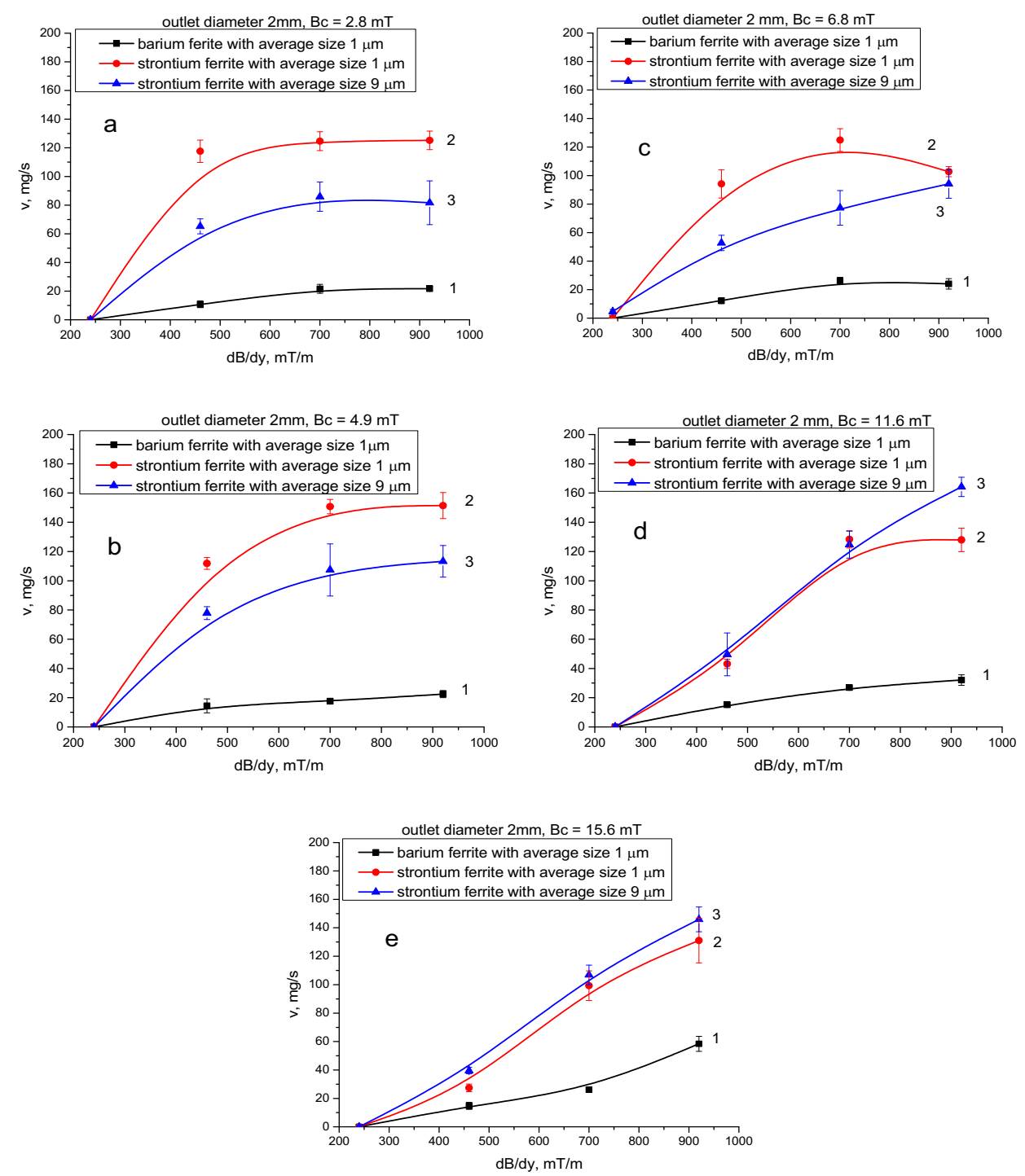

Fig. 2. Dependencies of efflux mass speed of barium hexaferrite powder (1) with average particle size $1 \mu \mathrm{m}$ and strontium hexaferrite powder with average particle size $1 \mu \mathrm{m}(2)$ and $9 \mu \mathrm{m}(3)$ through the outlet with diameter $2 \mathrm{~mm}$ from alternating field induction gradient at different values of constant field induction: a) $2.8 \mathrm{mT}$; b) $4.9 \mathrm{mT}$; c) $6.8 \mathrm{mT}$; d) $11.6 \mathrm{mT}$; e) $15.6 \mathrm{mT}$. 


\section{Results and discussion}

Fluidity regularities of barium hexaferrite powder with average particle size $1 \mu \mathrm{m}$ and strontium hexaferrite powders with average size 1 and $9 \mu \mathrm{m}$ through $2 \mathrm{~mm}$ diameter hopper outlet from electromagnetic effect parameters are presented on Fig. 2. As can be seen from the figure, there is no powder fluidity at variable magnetic field gradient values less than $230 \mathrm{mT} / \mathrm{m}$. Obviously, at these low gradient values processes of aggregate formations prevail processes of aggregate destruction. Further gradient increase leads to formation of magneto fluidized bed from powders under study, but the ratio of aggregation formation and aggregation destruction processes depends from individual powder properties.

The increase of variable field induction gradient to $700 \mathrm{mT} / \mathrm{m}$ at constant field induction values from $2.8 \mathrm{mT}$ to $15.6 \mathrm{mT}$ leads to increase of mass efflux speed. At increase of constant field induction from $2.8 \mathrm{mT}$ (Fig. 2a) to $4.9 \mathrm{mT}$ (Fig. 2b) all experimental dependencies have the same behaviour. In these field values the regularities are not affected by different average particle sizes. At constant field induction $6.8 \mathrm{mT}$ dependency curve of strontium hexaferrite with average particle size $1 \mu \mathrm{m}$ (curve 2 at Fig. 2c) has maximum at alternating field gradient $700 \mathrm{mT} / \mathrm{m}$. Further gradient increase to $920 \mathrm{mT} / \mathrm{m}$ decreases value of mass efflux speed by $22.2 \mathrm{mg} / \mathrm{s}$ while strontium hexaferrite with $9 \mu \mathrm{m}$ average size show increase by $16.9 \mathrm{mg} / \mathrm{s}$ (curve 3 at Fig. 2c).

Increase of constant field induction to $11.6 \mathrm{mT}$ leads to great change of mass efflux speed only for strontium hexaferrite with $9 \mu \mathrm{m}$ average particle size (Fig. 2d). For example, at alternating field induction gradient $920 \mathrm{mT} / \mathrm{m}$ increase of constant field induction from 6.8 to $11.6 \mathrm{mT}$ leads to increase of efflux speed of strontium hexaferrite powders with 1 and $9 \mu \mathrm{m}$ average size by 25.2 and $70.0 \mathrm{mg} / \mathrm{s}$ correspondingly. Increase of constant magnetic field induction to $15.6 \mathrm{mT}$ (Fig. 2e) allows to intensify efflux process of barium hexaferrite powder only.

Presented on Fig. 2 dependencies show, that fluidity of barium hexaferrite powder (curve 1) is significantly lower than of strontium hexaferrite (curves 2 and 3). For example, comparing fluidity of powders with average particle size $1 \mu \mathrm{m}$ (Fig. 2a) at $700 \mathrm{mT} / \mathrm{m}$ induction gradient and $2.8 \mathrm{mT}$ constant field induction, efflux speed of strontium hexaferrite is 5.8 times higher than of barium hexaferrite. Obtained results are consistent with previously developed representation about influence of alternating gradient and constant magnetic fields with mutually perpendicular induction lines on processes of structure formation in particulate ferromagnetic medium [21]. The effect of magnetic fields on particulate system is reduced to the change in magneto fluidized bed density and sizes of formed structured elements (aggregates) that influences on viscosity of the medium and powder fluidity through the outlet.

\section{Conclusion}

Powders of magnetic materials that don't have natural fluidity through the outlet obtain the fluidity at defined values of induction of constant and induction gradient of alternating magnetic fields that form magneto fluidized bed in particulate system with prevailing processes of particle aggregates destruction. Efflux speed of ferromagnetic powders through thin outlet grows at increase of alternating magnetic field gradient till defined for each powder value.

Fine strontium hexaferrite powder has higher fluidity in alternating gradient and constant magnetic fields than barium hexaferrite powder. Considering mentioned above results of barium and strontium hexaferrite powders stimulated fluidity formation, one can conclude that intensification of technological processes involving magnetohard powders fluidity is mostly defined by the magnetic effect parameters and magnetic fields configuration. 


\section{References}

1. J.M. Valverde, Springer Netherlands, 136 (2013)

2. L. Liu, X.D. Wang, X. Li, Int. J. of Minerals, Metallurgy and Materials, 24, 1021 (2017)

3. Q. Cao, Z. Li, Z. Wang, J. of Physics D: Applied Physics, 51, 1 (2018)

4. C. Dominik, H. Nubold, Icarus 157, 173 (2002)

5. W. Qin, J.He, J.Meng, Advanced Materials Research, 535-537, 1314 (2012)

6. M.J. Espin, J.M. Valverde, M.A.S. Quintanilla, J. of Rheology, 54, 719 (2010)

7. S.Dietmar, Powders and Bulk Solids, 512 (2008)

8. N.B. Uriev, Highly concentrated particulate systems, 320 (1980) (in Russian)

9. Yu.G. Dorofeev, Theory of metallic powders production and their moulding, 144 (1999) (in Russian)

10. J.Y. Hristov, 5th European Rheology Conference (Portoroz, Slovenia, 1998)

11. M. J. Espin, M. A. S. Quintanilla, J. M. Valverde, Chem. Eng. J., 277, 269 (2015)

12. M. J. Espin, J. M. Valverde, M. A. S. Quintanilla, J. of Fluid Mech., 732, 282 (2013)

13. E.I. Andrianov, Methods of study structural-mechanical characteristics of powder materials, 256 (1982) (in Russian)

14. B.E. Levin, Yu.D. Tretiyakov, L.M. Letyuk, Physico-chemical basics of production, properties and application of ferrites (1979) (in Russian)

15. S.I. Egorova, Magnetovibrating fluidization, 162 (2009) (in Russian)

16. I.N. Egorov, N.Ya. Egorov, MATEC Web Conf., 132, 03001 (2017)

17. I.N. Egorov, S.I. Egorova, A.I. Chernyi, Pat 022640 (EA) (2016)

18. D.D. Logvinenko, O.P. Shelyakov, Intensification of technological processes in devices with vortex layer (1976) (in Russian)

19. Yu.M. Vernigorov, I.N. Egorov, S.I. Egorova, Euro PM2005 Congress and Exhibition proceedings, 1, 451 (2005)

20. I.N. Egorov, S.I. Egorova, Russian Journal of Non_Ferrous Metals, 55, 371 (2014)

21. I.N. Egorov, Vestnik DGTU, 3, 44 (2012) (in Russian) 